\section{Ultra-Low Voltage \\ Scanning Electron Microscopy}

David C. Joy* and Carolyn S. Joy

University of Tennessee and *Oak Ridge National Laboratory

Although the benefits of operating the scanning electron microscope at low beam energies have been evident since the earliest days of the instrument, the successful utilization of the SEM under these conditions has required the development of high brightness field emission electron source, advanced lenses, and clean vacuums. As these technologies became available the level at which imaging became regarded as "low energy" has fallen from $10 \mathrm{keV}$, first to $5 \mathrm{keV}$, and more recently to $1 \mathrm{keV}$. At this energy state of the art, instruments can now provide an excellent balance between resolution - which becomes worse with decreasing energy - and desirable goals such as the minimization of sample charging and the reduction of macroscopic radiation damage - which tend to become more challenging as the energy is increased.

An interesting new opportunity is to perform imaging in the ultra-low energy region between $1 \mathrm{eV}$ and $500 \mathrm{eV}$. Over this energy range significant changes in the details of electron-solid interactions take place offering the chance of novel contrast modes, and the rapid fall in the electron beam range leads to the condition where the penetration of the incident beam into the sample is effectively limited to 1 or 2 nanometers. The practical problem is that of achieving useful levels of resolution and acceptable signal to noise ratios in the image. At energies below $1 \mathrm{keV}$ chromatic aberration dominates the probe formation in conventional instruments even when using an FEG source. However, the use of optimized retarding field optics essentially maintains chromatic aberration independent of landing energy down to very low values. ${ }^{1.2}$ Figure 1 shows an example of the performance that can be achieved on a commercial instrument - an Hitachi S-4500 modified to operate in this mode, in this case at $50 \mathrm{eV}$ landing energy. The resolution of the image is judged from edge sharpness and detail to be significantly better than $0.1 \mu \mathrm{m}$ and, from experimental observation, this performance is apparently limited by residual astigmatism caused by uncorrected sample charging rather than by fundamental aberrations in the probe forming optics. Comparable, if somewhat lower resolution, images have been achieved on this, and other FEG SEMs, at energies as low as 1 eV

At incident energies below $100 \mathrm{eV}$ it is necessary to describe the signal that is collected in terms that are rather different from those that are conventionally employed since the energy of both the backscattered and the secondary electrons are in the same range. Figure 2 shows a plot of the measured electron emission from tungsten in the incident energy range 1 $\mathrm{eV}$ to $10 \mathrm{KeV}$ where, for convenience, all of the emission in the low energy range is here referred to as secondary emission ${ }^{3}$. At high energies the SE

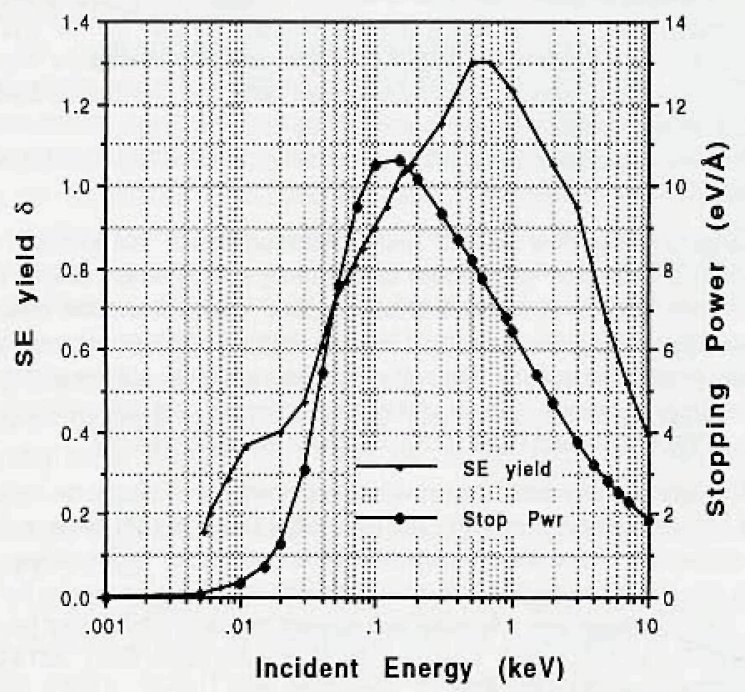

Figure 1. Image of diatom recorded at $50 \mathrm{eV}$ in modified Hitachi S-4500 FEGSEM signal shows the usual rapid rise as the incident energy falls, reaching a maximum at about $600 \mathrm{eV}$. As the energy is further reduced the signal is seen to fall once more such that at an incident energy of $10 \mathrm{eV}$ the "secondary signal" once again has about the same magnitude as the SE yield at $10 \mathrm{keV}$. In the energy range of interest, therefore, signal levels are comparable with those found at more usual accelerating voltages. It is interesting to compare this yield profile with the variation of electron stopping power in the material also plotted, in units of eV/A, on figure $2^{3}$. Although the respective maxim of the two curves are displaced in energy it is evident that Bethe's hypothesis that electron yield is proportional to the stopping power is essentially correct. At incident energies above the stopping power maximum additional SE production (i.e., SEII components) results from the backscattered signal. Although experimental data is limited it is probable that these levels of signal production are typical for most materials of interest. The form of image contrast is unlike that at higher energies since the beam penetration depth is less than the SE escape range. There is, thus, no topographic contrast, although shadowing can often be observed. The most common mode of contrast formation is associated with surface charging. The electron range is of the same order as native oxide film thickness on many metals and semiconductors, and the yield is $<1$. Hence negative charging occurs which may result in the formation of localized electron mirrors. At the lowest energies, below $30 \mathrm{eV}$, interesting anomalous effects have been observed which may be associated with the increase of inelastic mean free path length in this regime.

Signal to noise limitations are important at these low energies as the gun brightness is much reduced compared to normal operation. An acceptable quality image is achieved by slow-speed scanning, but TV rate imaging is not presently possible. The efficiency of the detection system is clearly of importance in this case. The best arrangement uses the retarding field to accelerate the emitted 'secondaries' after they leave the specimen and before they enter the collection field of the detector. This ensures adequate quantum efficiency and guarantees symmetrical collection even if the detector is physically offset. Future development of instrumentation for ultra-low energy SEM would ideally include both improved detection systems and perhaps the use of nano-tip field emission sources which offer 100 to $1000 \mathrm{x}$ more brightness than conventional FEG sources. ${ }^{4}$ Initial work is now proceeding in these directions. ${ }^{5}$

1. I. Mullerova and L. Frank, SCANNING 15, (1993), 193

2. A.D. Brodie, J. Vac. Sci. Technol. B12, (1994), 3489

3. D.C. Joy, SCANNINGG 17, (1995), 270. A complete copy of this data base is available from the author. The BS and SE data may also be accessed at http:/Www. nsctoronto com/ nissei-sangyo

4. J.C. H. Spence et al., J Vac Sci., A12, (1994), 542

5. Oak Ridge National Laboratory is operated by Lockheed Martin Energy Research Corp. for the U.S. Department of Energy under contract number DE-AC05-960R22464.

Reprinted with permission from Microscopy and Microanalysis 1996.

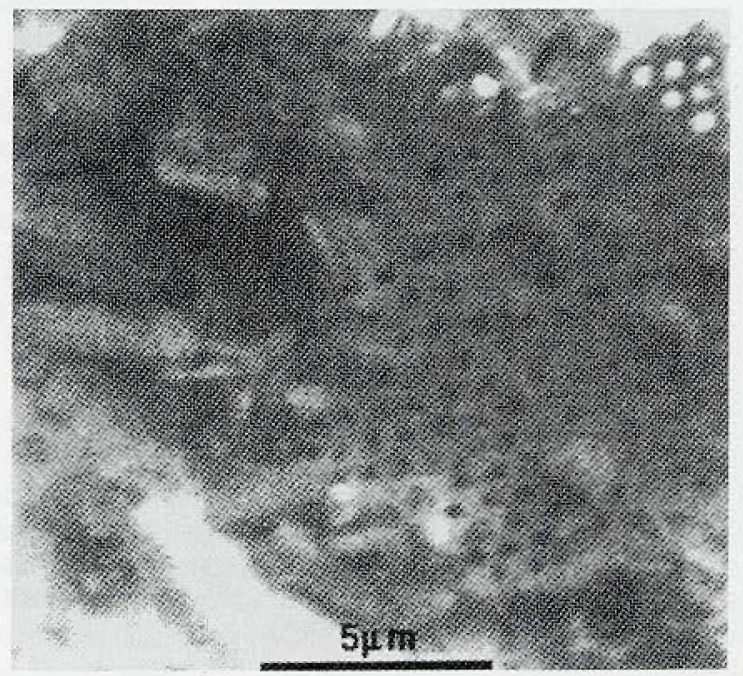

Figure 2. Variation with energy of SE yield and electron stopping power (in eV/A) for tungsten. 


\section{Nothing tricky, nothing complex. Just rugged, reliable conventional SEMs.}

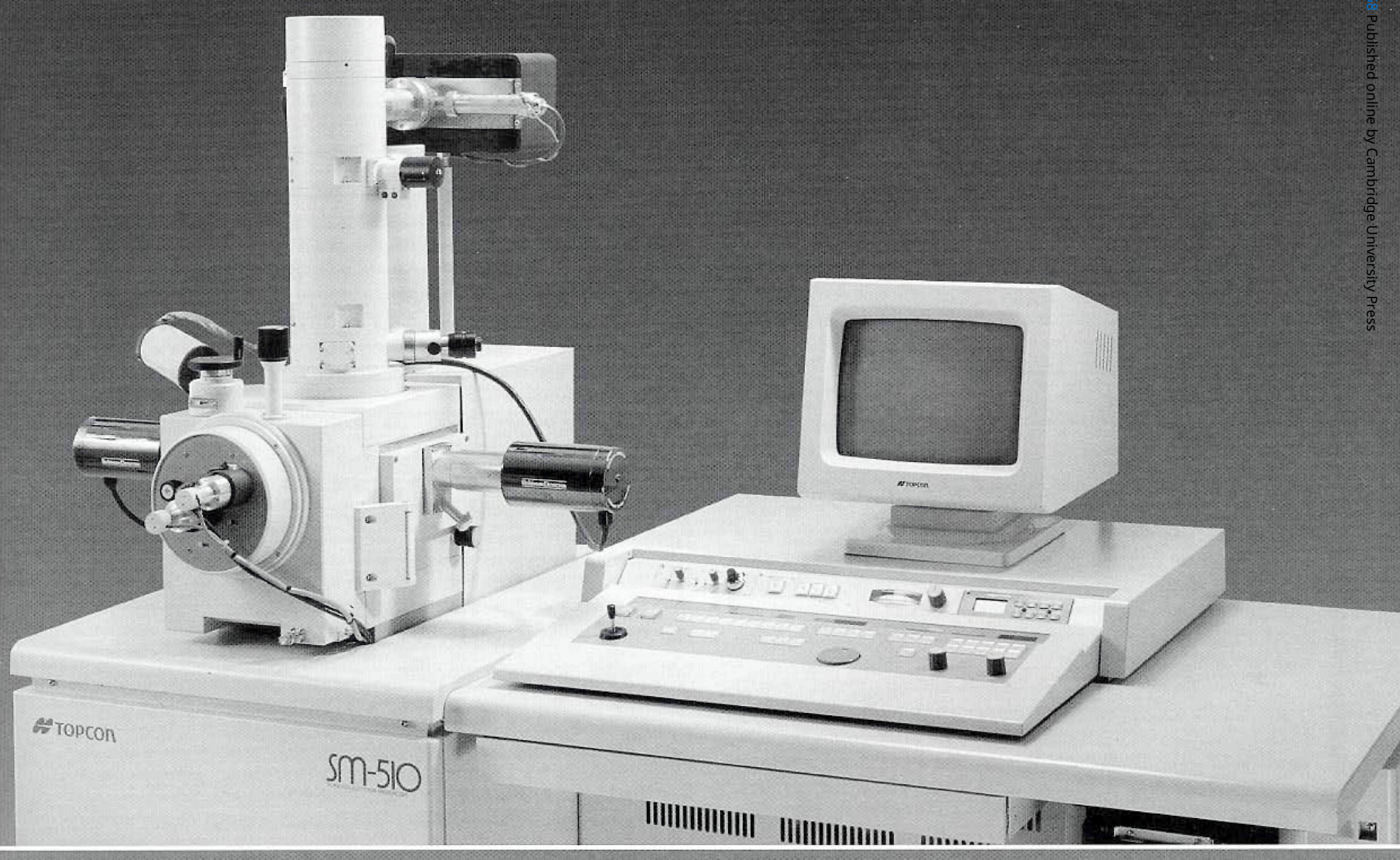

The worlds of science and industry get more complicated. No wonder that tried and true instruments, like Topcon's conventional SEMs, take on greater value.

In fact, $93 \%$ of our users say Topcon SEMs are today's greatest value. Why?

Because they are rugged, reliable, dependable, with nothing tricky or hard to learn.

For 20 years, our SEMs have been dedicated to making your world easier. They take scanning electron microscopy out of the hands of the technical elite... and put all their power in your hands instantly.

Want to know more? Call and tell us about your applications. Toll free of course. Ask for our new literature. 1-800-538-6850.

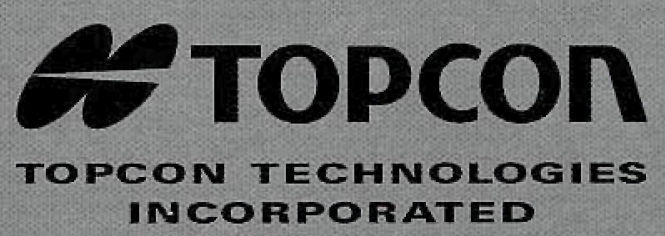

